\title{
A DIRECT MEASUREMENT OF THE TOTAL GAS COLUMN DENSITY IN ORION KL
}

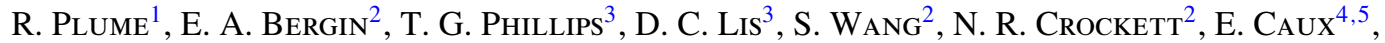 \\ C. Сомito ${ }^{6}$, P. F. GoldDsmith ${ }^{7}$, AND P. SCHILKE ${ }^{6,8}$ \\ ${ }^{1}$ Department of Physics \& Astronomy and the Institute for Space Imaging Sciences, University of Calgary, \\ Calgary, AB T2N 1N4, Canada; plume@ ras.ucalgary.ca \\ ${ }^{2}$ Department of Astronomy, University of Michigan, 500 Church Street, Ann Arbor, MI 48109, USA \\ ${ }^{3}$ California Institute of Technology, Cahill Center for Astronomy and Astrophysics 301-17, Pasadena, CA 91125, USA \\ ${ }^{4}$ Université de Toulouse, UPS-OMP, IRAP, Toulouse, France \\ ${ }^{5}$ CNRS, IRAP, 9 Av. colonel Roche, BP 44346, F-31028 Toulouse cedex 4, France \\ ${ }^{6}$ Max-Planck-Institut für Radioastronomie, Auf dem Hügel 69, 53121 Bonn, Germany \\ ${ }^{7}$ Jet Propulsion Laboratory, Caltech, Pasadena, CA 91109, USA \\ ${ }^{8}$ I. Physikalisches Institut, Universität zu Köln, Zülpicher Str. 77, 50937 Köln, Germany \\ Received 2011 May 5; accepted 2011 September 11; published 2011 December 9
}

\begin{abstract}
The large number of high-J lines of $\mathrm{C}^{18} \mathrm{O}$ available via the Herschel Space Observatory provide an unprecedented ability to model the total $\mathrm{CO}$ column density in hot cores. Using the emission from all the observed lines (up to $J=15-14$ ), we sum the column densities in each individual level to obtain the total column after correcting for the population in the unobserved states. With additional knowledge of source size, $V_{\mathrm{LSR}}$, and line width, and both local thermodynamic equilibrium (LTE) and non-LTE modeling, we have determined the total $\mathrm{C}^{18} \mathrm{O}$ column densities in the Extended Ridge, Outflow/Plateau, Compact Ridge, and Hot Core components of Orion KL to be $1.4 \times 10^{16} \mathrm{~cm}^{-2}, 3.5 \times 10^{16} \mathrm{~cm}^{-2}, 2.2 \times 10^{16} \mathrm{~cm}^{-2}$, and $6.2 \times 10^{16} \mathrm{~cm}^{-2}$, respectively. We also find that the $\mathrm{C}^{18} \mathrm{O} / \mathrm{C}^{17} \mathrm{O}$ abundance ratio varies from 1.7 in the Outflow/Plateau, 2.3 in the Extended Ridge, 3.0 in the Hot Core, and to 4.1 in the Compact Ridge. This is in agreement with models in which regions with higher ultraviolet radiation fields selectively dissociate $\mathrm{C}^{17} \mathrm{O}$, although care must be taken when interpreting these numbers due to the size of the uncertainties in the $\mathrm{C}^{18} \mathrm{O} / \mathrm{C}^{17} \mathrm{O}$ abundance ratio.
\end{abstract}

Key words: ISM: abundances - ISM: individual objects (Orion KL) - ISM: molecules

Online-only material: color figures

\section{INTRODUCTION}

The total $\mathrm{H}_{2}$ column density is a fundamental quantity used to determine a variety of properties that are important for studies of the interstellar medium (ISM) and star formation, such as chemical abundances, the importance of (self-)shielding from radiation fields, cloud mass, and the onset of molecular freezeout onto dust grains. However, $\mathrm{H}_{2}$ itself cannot be observed at typical temperatures $(\sim 10-30 \mathrm{~K})$ found in the cold dense ISM where stars are born. This is because $\mathrm{H}_{2}$ is a very light molecule with widely spaced rotational transitions $\left(\Delta E_{u} / k=510 \mathrm{~K}\right.$ for the ground state) and only has weak quadrupole transitions $\left(\mathrm{H}_{2}\right.$ has no dipole). Therefore, one often turns to thermal emission from dust or spectral line emission from $\mathrm{CO}$ as a proxy for the $\mathrm{H}_{2}$ column. Unfortunately, the presence of sharp excitation gradients (density/temperature) toward massive star-forming regions severely complicates the determination of total column density from the dust emission (e.g., Goldsmith et al. 1997; Shirley et al. 2011) and one also has to rely on an uncertain but canonical dust-to-gas conversion ratio. In addition, since $\mathrm{CO}$ is often optically thick, especially toward the highest column density regions in molecular clouds, where stars are born, the total $\mathrm{H}_{2}$ column density is often poorly constrained. An additional complication arises due to the fact that $\mathrm{CO}$ is depleted onto dust grains in cold $(T<20 \mathrm{~K})$ dense clumps (e.g., Bergin \& Tafalla 2007; Acharyya et al. 2007). However, in warm $(T \gg 20 \mathrm{~K})$ gas surrounding massive or even low-mass protostars (so-called Hot Cores and Hot Corinos), depletion is not a factor and the rarer isotopologues $\left({ }^{13} \mathrm{CO}, \mathrm{C}^{18} \mathrm{O}\right.$, and $\left.\mathrm{C}^{17} \mathrm{O}\right)$ can be used as total column density tracers.
The method we have isolated draws upon that suggested by Goldsmith et al. (1997) and offers an unprecedented opportunity to derive this fundamental quantity in a model independent fashion. The basic idea is simple and elegant. For an optically thin line the observed integrated emission is proportional to the column density in the upper state $\left(N_{u}\right)$. This quantity can be derived without any assumptions regarding density or temperature. If you observe enough transitions of $\mathrm{C}^{18} \mathrm{O}$, one can simply estimate the total column from $N_{\text {tot }}=f_{c} \sum N_{u}$, where $f_{c}$ corrects for the missing population. Typically, one observes low- $J\left(J_{u}<3\right)$ lines from the ground and then assumes that all the rotational levels are populated according to some assumed partition function (often based on the Boltzman equation with a single excitation temperature). In high-mass star-forming regions in the presence of Hot Cores, the temperatures can be $>100 \mathrm{~K}$ and the densities can be $>10^{6} \mathrm{~cm}^{-3}$. Under these conditions, the higher- $J$ states can be significantly populated and an estimate of the total column density based on only a few low-energy transitions can be seriously in error. With the Heterodyne Instrument for the Far Infrared (HIFI) instrument on board the Herschel Space Observatory, we have access to $>6$ $\mathrm{C}^{18} \mathrm{O}$ transitions with $J \geqslant 5$ (+ ground-based low- $J$ lines), and under these conditions the correction factor $f_{c}$ becomes quite small.

In this paper we demonstrate the utility of the Herschel/ HIFI observations of $\mathrm{C}^{18} \mathrm{O}$ to obtain the total molecular column densities in Orion KL. Orion KL has been the target of numerous millimeter and submillimeter line surveys (e.g., Blake et al. 1987; Comito et al. 2005; Persson et al. 2007) that have revealed and confirmed the presence of several distinct components 
along the line of sight. The Extended Ridge is a fairly cool $\left(T_{K} \approx 40 \mathrm{~K}\right)$, quiescent $\left(V_{\mathrm{LSR}} \approx 8 \mathrm{~km} \mathrm{~s}^{-1}\right.$ in the south to $\approx 10 \mathrm{~km} \mathrm{~s}^{-1}$ in the north with $\Delta V_{\mathrm{FWHM}} \approx 3-5 \mathrm{~km} \mathrm{~s}^{-1}$ ), extended $\left(\approx 180^{\prime \prime}\right)$ ridge of gas. The Outflow/Plateau is warmer $\left(T_{K} \approx 100-150 \mathrm{~K}\right)$ and smaller $\left(\approx 20^{\prime \prime}-70^{\prime \prime}\right)$ than the Extended Ridge and has two distinct kinematic components-a highvelocity component $\left(\Delta V_{\mathrm{FWHM}}>30 \mathrm{~km} \mathrm{~s}^{-1}\right)$ centered slightly north of IRc 2 (Wright et al. 1983) and a low-velocity component $\Delta V_{\text {FWHM }} \gtrsim 18 \mathrm{~km} \mathrm{~s}^{-1}$ ) which is centered on IRc 2 itself (Plambeck et al. 1982). In this paper we focus on the lowvelocity component since, in our data, we see little evidence for $\mathrm{C}^{18} \mathrm{O}$ or $\mathrm{C}^{17} \mathrm{O}$ emission from the high-velocity component. The Compact Ridge has similar kinematics to the Extended Ridge $\left(\Delta V_{\mathrm{FWHM}} \approx 3-5 \mathrm{~km} \mathrm{~s}^{-1}\right.$ and $\left.V_{\mathrm{LSR}} \approx 8-10 \mathrm{~km} \mathrm{~s}^{-1}\right)$ but is spatially distinct, smaller $\left(25^{\prime \prime}\right)$, and hotter $\left(T_{K} \sim 125 \mathrm{~K}\right.$; Bergin et al. 1994). The Hot Core is the smallest and hottest of all the components $\left(10^{\prime \prime}\right.$ and $\approx 150 \mathrm{~K}$, respectively), and is also kinematically distinct from the other components-with $V_{\mathrm{LSR}}=3-4 \mathrm{~km} \mathrm{~s}^{-1}$ and $\Delta V_{\mathrm{FWHM}} \approx 9 \mathrm{~km} \mathrm{~s}^{-1}$. The Hot Core is centered $\approx 2^{\prime \prime}$ from IRc 2 and is heated internally by young massive protostars.

In Section 2 we describe our Herschel observations, while in Section 3 we present and discuss our modeling and results, including a detailed discussion of the uncertainties on the derived columns, which we estimate to be generally less than $25 \%$. We summarize our results in Section 4.

\section{OBSERVATIONS}

The observations presented here were taken as part of the Herschel/HIFI Observations of EXtraOrdinary Sources (HEXOS) Guaranteed Time Key Project on the Herschel Space Observatory (Bergin et al. 2010). A detailed description of these observations is given by Bergin et al. (2010) but, in summary, the observations were carried out using the dual beam switch mode pointed toward the Orion Hot Core at $\alpha_{\mathrm{J} 2000}=5^{\mathrm{h}} 35^{\mathrm{m}} 14^{\mathrm{s}} .3$ and $\delta_{\mathrm{J} 2000}=-5^{\circ} 22^{\prime} 36^{\prime \prime}$.7. We used the Wide Band Spectrometer providing a spectral resolution of $1.1 \mathrm{MHz}$ over a $4 \mathrm{GHz}$ intermediate frequency bandwidth and only use the data from the horizontal polarization (due to excess noise seen in the vertical polarization data). We applied the standard HIFI deconvolution using the doDeconvolution task within the Herschel Interactive Processing Environment (Version 5.0) and all data presented here are deconvolved single sideband spectra. In addition, the standard HIFI beam efficiencies $\left(\eta_{\mathrm{mb}}\right)$ have been applied so that all data are presented and modeled as main beam temperatures where $T_{\mathrm{mb}}=T_{A}^{*} / \eta_{\mathrm{mb}}$. The HIFI $\mathrm{C}^{18} \mathrm{O}$ and $\mathrm{C}^{17} \mathrm{O}$ lines available for our analysis are listed in Table 1 . Herschel beam sizes range from $\approx 40^{\prime \prime}$ in Band $1 \mathrm{~A}$ to $13^{\prime \prime}$ in Band $6 \mathrm{~B}$ and are also listed in Table 1 along with the velocity resolution of the observations (in some cases smoothed to improve $\mathrm{S} / \mathrm{N}$ ).

In addition to the Herschel/HIFI observations, we have ground-based observations of $\mathrm{C}^{18} \mathrm{O} \quad 3-2(329.330 \mathrm{GHz})$, $\mathrm{C}^{18} \mathrm{O} 2-1(219.560 \mathrm{GHz})$, and $\mathrm{C}^{17} \mathrm{O} 2-1(224.714 \mathrm{GHz})$ at the same position, using the $10.4 \mathrm{~m}$ Caltech Submillimeter Observatory (CSO) on Mauna Kea, Hawaii. These observations were taken in 2009 October and November using a spectral resolution of $0.488 \mathrm{MHz}\left(\sim 0.4 \mathrm{~km} \mathrm{~s}^{-1}\right)$. The system temperature for these observations was $1000 \mathrm{~K}$ for the 3-2 observations and $\sim 400 \mathrm{~K}$ for the $2-1$ observations. The $\mathrm{C}^{18} \mathrm{O} 3-2$ data have been corrected to the $T_{\mathrm{mb}}$ scale using a main beam efficiency of 0.75 . The main beam efficiencies of $\mathrm{C}^{18} \mathrm{O}$ and $\mathrm{C}^{17} \mathrm{O} 2-1$ are 0.77 and 0.70 , respectively. The beam sizes of the $\mathrm{CSO}$ at $330 \mathrm{GHz}$ are
Table 1

Line Parameters

\begin{tabular}{lcccccc}
\hline \hline HIFI Band & Line & $\begin{array}{c}\text { Freq } \\
(\mathrm{GHz})\end{array}$ & $\begin{array}{c}\text { rms } \\
(\mathrm{K})\end{array}$ & $\eta_{\mathrm{mb}}$ & $\theta_{\mathrm{mb}}\left(^{\prime \prime}\right)$ & $\begin{array}{c}\Delta V_{\mathrm{FWHM}} \\
\left(\mathrm{km} \mathrm{s}^{-1}\right)\end{array}$ \\
\hline Band 1A & $\mathrm{C}^{18} \mathrm{O} 5-4$ & 548.831 & 0.05 & 0.68 & 40 & 0.29 \\
Band 2B & $\mathrm{C}^{18} \mathrm{O}-6$ & 768.251 & 0.10 & 0.68 & 28 & 0.20 \\
& $\mathrm{C}^{17} \mathrm{O}-6$ & 786.280 & 0.09 & 0.68 & 27 & 0.20 \\
Band 3B & $\mathrm{C}^{18} \mathrm{O} 8-7$ & 877.922 & 0.10 & 0.68 & 24 & 0.16 \\
& $\mathrm{C}^{17} \mathrm{O} 8-7$ & 898.523 & 0.05 & 0.68 & 24 & 0.16 \\
Band 4A & $\mathrm{C}^{18} \mathrm{O} 9-8$ & 987.560 & 0.07 & 0.67 & 22 & 0.15 \\
& $\mathrm{C}^{17} \mathrm{O} 9-8$ & 1010.731 & 0.08 & 0.67 & 21 & 0.15 \\
Band 5A & $\mathrm{C}^{18} \mathrm{O} 11-10$ & 1206.725 & 0.13 & 0.66 & 18 & 0.13 \\
& $\mathrm{C}^{17} \mathrm{O} 10-9$ & 1122.902 & 0.12 & 0.66 & 19 & 0.13 \\
& $\mathrm{C}^{17} \mathrm{O} 11-10$ & 1235.031 & 0.16 & 0.66 & 17 & 0.13 \\
Band 6A & $\mathrm{C}^{17} \mathrm{O} 13-12$ & 1459.147 & 0.74 & 0.65 & 15 & 0.41 \\
Band 6B & $\mathrm{C}^{18} \mathrm{O} 15-14$ & 1644.495 & 0.59 & 0.64 & 13 & 0.73 \\
& $\mathrm{C}^{17} \mathrm{O} 15-14$ & 1683.045 & 0.60 & 0.64 & 13 & 0.73 \\
Band 7B & $\mathrm{C}^{18} \mathrm{O} 17-16$ & 1863.037 & 0.42 & 0.63 & 12 & 0.65 \\
& $\mathrm{C}^{17} \mathrm{O} 16-15$ & 1794.902 & 0.62 & 0.63 & 12 & 0.65 \\
\hline
\end{tabular}

$220 \mathrm{GHz}$ are $\approx 21^{\prime \prime}$ and $32^{\prime \prime}$, respectively and so are moderately well-matched to the Herschel/HIFI beam sizes.

\section{RESULTS AND DISCUSSION}

\subsection{Modeling the $\mathrm{C}^{18} \mathrm{O}$ Line Emission}

To obtain the total $\mathrm{C}^{18} \mathrm{O}$ column density from a single transition, a typical assumption is that the emission is optically thin and in local thermodynamic equilibrium (LTE). In this case, the integrated intensity is linearly related to the column density in the upper state $\left(N_{u}\right)$. The total column density $\left(N_{\text {tot }}\right)$ can be estimated with a partition function based on the Boltzman equation and a single excitation temperature. Alternatively, one can use a more sophisticated non-LTE modeling technique in which the coupled equations of radiative transfer and detailed balance are solved together. Here it is typical to simply adjust the total column density until the model integrated intensity matches the observations. Large velocity gradient (LVG) and other publicly available codes such as RADEX (van der Tak et al. 2007) are often employed for this task.

With observations of multiple transitions, however, the situation becomes a little more complicated. The most direct method to obtain $N_{\text {tot }}$ would be to observe every possible transition, calculate the $N_{u}$ in each upper state, and simply sum them all to obtain the column in all levels. This is quite powerful because it is a temperature and excitation independent measure of the total column. In practice, however, this is difficult to do due to high atmospheric opacity at many wavelengths and finite telescope time. A more typical case is the observation of just a few transitions from a single molecule which can be compared to a grid of models (each with a different $N_{\text {tot }}$ ) to identify the model which gives the best fit to the observed line intensities via a $\chi^{2}$ minimization technique (see, for example, Plume et al. 1997). The "best fit," however, is not always a good fit, since a single $N_{\text {tot }}$ solution may not match the observed line intensities for all transitions.

With the HIFI instrument on board the Herschel Space Observatory, however, we have access to seven high-frequency $\mathrm{C}^{18} \mathrm{O}$ transitions (see Table 1) and, combined with lower frequency ground-based observations, have a unique opportunity to directly calculate the total $\mathrm{C}^{18} \mathrm{O}$ column density in Orion KL. Rather than using a $\chi^{2}$ minimization technique, we have taken a slightly different approach based on a technique sug- 

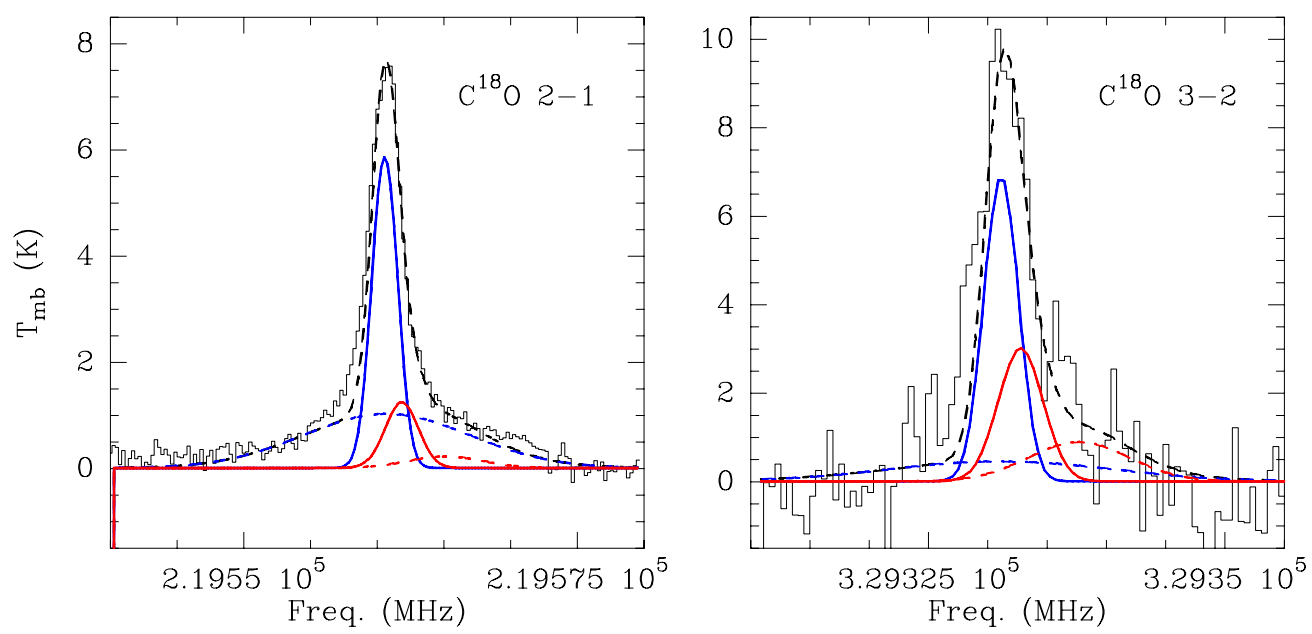

Figure 1. CSO spectrum (histogram) and result of LTE modeling of the $\mathrm{C}^{18} \mathrm{O} J=2-1(219.56 \mathrm{GHz})$ and $3-2(329.330 \mathrm{GHz})$ in Orion KL. Results are shown for the Extended Ridge (solid blue), Outflow/Plateau (dashed blue), Compact Ridge (solid red), Hot Core (dashed red), and for the sum of all four components (dashed black).

(A color version of this figure is available in the online journal.)

Table 2

Line-of-sight Components toward Orion KL

\begin{tabular}{lrrcrc}
\hline \hline Component & $\begin{array}{c}T \\
(\mathrm{~K})\end{array}$ & $\begin{array}{c}\text { Size } \\
\left({ }^{\prime \prime}\right)\end{array}$ & $\begin{array}{c}V_{\mathrm{LSR}} \\
\left(\mathrm{km} \mathrm{s}^{-1}\right)\end{array}$ & $\begin{array}{c}\Delta V_{\text {FWHM }} \\
\left(\mathrm{km} \mathrm{s}^{-1}\right)\end{array}$ & $\begin{array}{c}n \\
\left(\mathrm{~cm}^{-3}\right)\end{array}$ \\
\hline Extended Ridge & 40 & 180 & $8-10$ & 3.0 & $10^{5}$ \\
Outflow/Plateau & 100 & 60 & $8-10$ & 20.0 & $10^{6}$ \\
Compact Ridge & 125 & 25 & $8-10$ & 4.0 & $10^{6}$ \\
Hot Core & 150 & 10 & $3-4$ & 9.0 & $10^{7}$ \\
\hline
\end{tabular}

Note. Information obtained from Blake et al. (1987), Genzel \& Stutzki (1989), and Bergin et al. (1994).

gested by Goldsmith et al. (1997). We derive $N_{u}$ for each of the nine observed transitions (seven Herschel lines and two from the CSO). Orion KL is known to have different physical and kinematic components which, combined, produce the overall observed line profile. The physical parameters used to describe and model these components are given in Table 2. For each transition we vary the upper state $\mathrm{C}^{18} \mathrm{O}$ column density $\left(N_{u}\right)$ for each of the four components until we produce the best overall line profile. The spectra and model fits are shown in Figures 1 and 2 .

The spectral modeling was done in two ways. We first adopt LTE, as implemented within WEEDS (Maret et al. 2011; part of the CLASS data reduction and analysis software package) and, second, we used the RADEX non-LTE code (van der Tak et al. 2007), as implemented within the CASSIS data analysis package $^{9}$. Both approaches model the line intensity and profile taking into account the beam filling factor by coupling the source size to the telescope beam size as a function of frequency, the kinetic temperature of the gas, the source velocity, and the line FWHM. The RADEX model also requires the source density.

When modeling the $\mathrm{C}^{18} \mathrm{O}$ emission in Orion $\mathrm{KL}$, great care must be taken to identify other molecular species that may be contaminating the observed spectral line profile. To do so, we used the Cologne Database for Molecular Spectroscopy (Müller et al. 2001, 2005) and Jet Propulsion Laboratory (JPL; Pickett et al. 1998) databases. In most cases, the spectral line

\footnotetext{
9 CASSIS has been developed by IRAP-UPS-OMP/CNRS
} (http://cassis.cesr.fr). profile was well fit solely by $\mathrm{C}^{18} \mathrm{O}$ emission from the four components of Orion KL. However, in some cases, additional species contributed to the observed profile at frequencies offset from those of $\mathrm{C}^{18} \mathrm{O}$ and, in a few specific cases, to the emission at similar frequencies to $\mathrm{C}^{18} \mathrm{O}$. In these latter cases especially, the contributions from each of the contaminating species had to be carefully modeled in order to extract the true contribution from $\mathrm{C}^{18} \mathrm{O}$.

In the case of $\mathrm{C}^{18} \mathrm{O} 2-1$ and 3-2 (Figure 1) and 5-4 (Figure 2, top left panel), no other lines contribute to the emission and the spectral line profile was modeled by $\mathrm{C}^{18} \mathrm{O}$ alone. For $\mathrm{C}^{18} \mathrm{O} 7-6$ (Figure 2, top right panel), while there are no other molecular species that contribute to the emission at the frequencies of $\mathrm{C}^{18} \mathrm{O}$, there are additional features at 768.205 and $768.315 \mathrm{GHz}$ that have been identified as likely arising from $\mathrm{CH}_{3} \mathrm{OH}$ and ${ }^{13} \mathrm{CH}_{3} \mathrm{OH}$, respectively. These are shown as contributing to the overall model profile (the black dashed line) but not to the colored lines which represent only the $\mathrm{C}^{18} \mathrm{O}$ emission from the four line-of-sight components. In the $\mathrm{C}^{18} \mathrm{O} 8-7$ panel (Figure 2, middle left), the feature at $877.97 \mathrm{GHz}$ has been identified as arising from numerous transitions of $\mathrm{CH}_{3} \mathrm{OCH}_{3}$. No other species contribute to the emission at the $\mathrm{C}^{18} \mathrm{O}$ frequencies. While $\mathrm{C}^{18} \mathrm{O} 9-8$ (Figure 2, middle right) seems, at first glance, to have no other spectral components, there is emission from ${ }^{33} \mathrm{SO}_{2}$ that occurs at similar frequency to that of $\mathrm{C}^{18} \mathrm{O}$ and thus contributes to the overall emission. Sulphur bearing species are thought to be tracers of outflows from young stellar objects and, therefore, using $\mathrm{N}\left({ }^{34} \mathrm{SO}_{2}\right)=5.4 \times 10^{16} \mathrm{~cm}^{-2}$ and ${ }^{33} \mathrm{SO} /{ }^{34} \mathrm{SO}=$ 0.2 (Persson et al. 2007; Comito et al. 2005) we model the ${ }^{33} \mathrm{SO}_{2}$ emission as arising from the Outflow/Plateau component with a total column density of $10^{16} \mathrm{~cm}^{-2}$. For $\mathrm{C}^{18} \mathrm{O} 11-10$ (Figure 2, bottom left) there is a small amount of $\mathrm{SO}_{2}$ emission that contributes to the overall profile (and to a small feature at $1206.58 \mathrm{GHz}$ ), but not at the frequencies corresponding to $\mathrm{C}^{18} \mathrm{O}$. The notable feature at 1206.79 is most likely due to $\mathrm{NHD}_{2}$. Finally, in the $\mathrm{C}^{18} \mathrm{O}$ 15-14 panel (Figure 2, bottom right), there are a number of additional spectral features (most notably the one at $1644.4 \mathrm{GHz}$ ) which all arise from $\mathrm{CH}_{3} \mathrm{OH}$. Modeling $\mathrm{CH}_{3} \mathrm{OH}$ as arising from the Compact Ridge with a column density of $8 \times 10^{16} \mathrm{~cm}^{-2}$ (which is a bit higher than the $5 \times 10^{16} \mathrm{~cm}^{-2}$ found by Comito et al. 2005) fits all the observed components and also shows that this species contributes the 

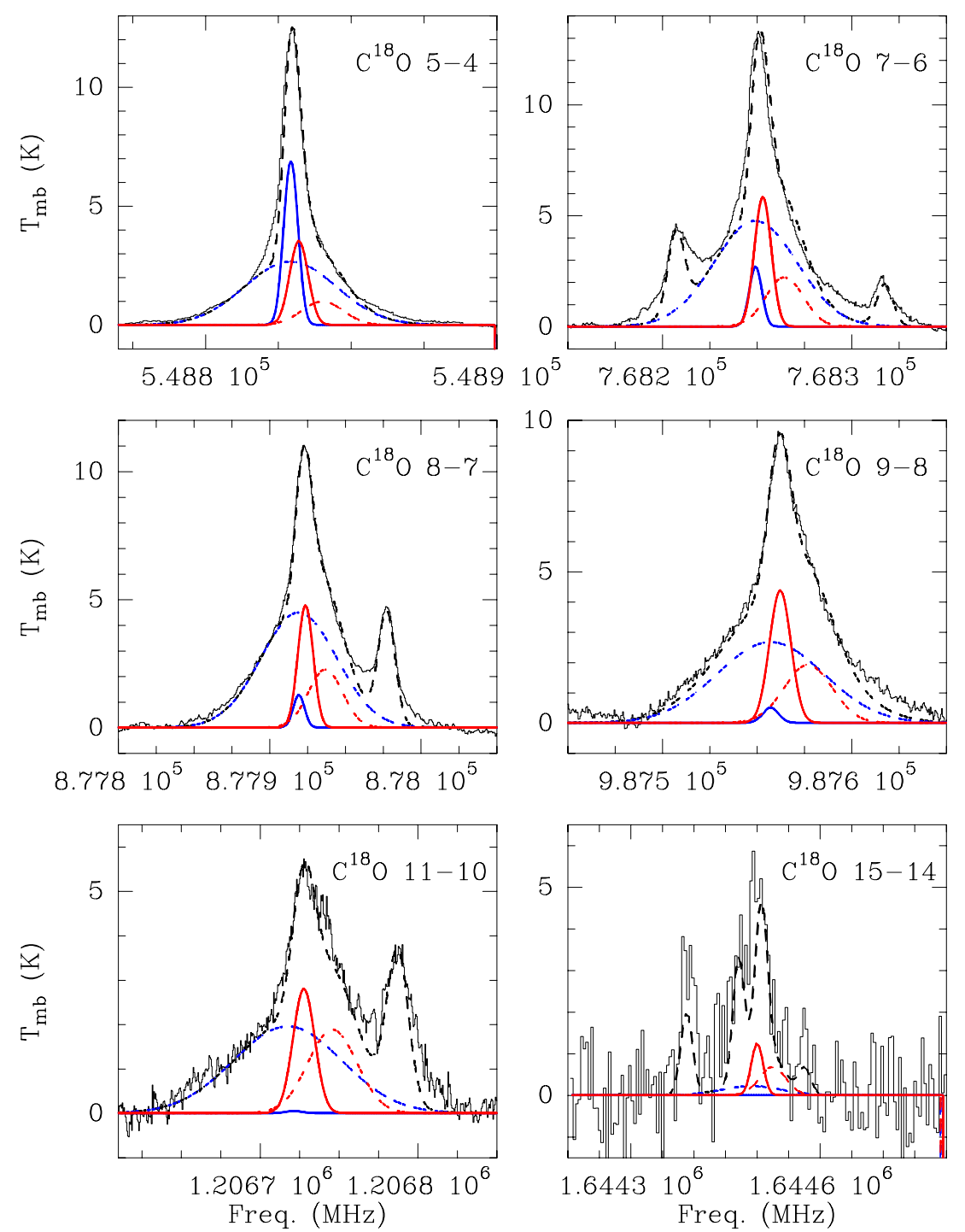

Figure 2. Herschel spectra (histograms) and results of LTE modeling of the $\mathrm{C}^{18} \mathrm{O} J=5-4(548.831 \mathrm{GHz}), J=7-6(768.251 \mathrm{GHz}), J=8-7(877.922 \mathrm{GHz}), J=$ 9-8 (987.560 GHz), $J=11-10(1206.725 \mathrm{GHz})$, and $J=15-14(1644.495 \mathrm{GHz})$ transitions in Orion KL. Results are shown for the Extended Ridge (solid blue), Outflow/Plateau (dashed blue), Compact Ridge (solid red), Hot Core (dashed red), and for the sum of all four components, including emission from species other than $\mathrm{C}^{18} \mathrm{O}$ (dashed black). For $\mathrm{C}^{18} \mathrm{O} 7-6$ the features at 768.205 and $768.315 \mathrm{GHz}$ are $\mathrm{CH}_{3} \mathrm{OH}$ and ${ }^{13} \mathrm{CH}_{3} \mathrm{OH}$, respectively. In the $\mathrm{C}^{18} \mathrm{O} 8-7$ panel, the feature at $877.97 \mathrm{GHz}$ is due to numerous transitions of $\mathrm{CH}_{3} \mathrm{OCH}_{3}$. For $\mathrm{C}^{18} \mathrm{O} 11-10$ there is $\mathrm{SO}_{2}$ emission that contributes slightly to the overall profile (and to a feature at $1206.58 \mathrm{GHz}$ ). The additional feature at 1206.79 is most likely due to $\mathrm{NHD}_{2}$. In the $\mathrm{C}^{18} \mathrm{O} 15-14$ panel, the feature at $1644.4 \mathrm{GHz}^{-15}$ probably $\mathrm{CH}_{3} \mathrm{OH}$ which also contributes the majority of the emission at the $\mathrm{C}^{18} \mathrm{O}$ frequency.

(A color version of this figure is available in the online journal.)

bulk of the emission at this frequency. Additional emission at $1644.4 \mathrm{GHz}$ may be contributed by $\mathrm{D}_{2}^{34} \mathrm{~S}$ which is not modeled in this figure. $\mathrm{C}^{18} \mathrm{O}$ itself is a minor contributor to the overall spectral line profile at $1644 \mathrm{GHz}$. The $\mathrm{C}^{18} \mathrm{O} 17-16$ spectrum is not shown since there was no detectable emission at this frequency.

From the modeling process described above, we obtain $N_{u}$ for each transition in each line-of-sight component. We then estimate the total column from $N_{\text {tot }}=f_{c} \sum N_{u}$, where $f_{c}$ corrects for the missing population. Figure 3 shows a calculation of $f_{c}$ for our observed $\mathrm{C}^{18} \mathrm{O}$ transitions. This calculation is performed using an LVG model with a total column density of $5 \times 10^{16} \mathrm{~cm}^{-2}$ (adjusting the column density between $10^{16}$ and $10^{17} \mathrm{~cm}^{-2}$ changes $f_{c}$ by less than $1 \%$ ), densities of $10^{5}$, $10^{6}$, and $10^{7} \mathrm{~cm}^{-3}$, and temperatures spanning $10-400 \mathrm{~K}$. At each temperature the code solves for the populations in the first 18 rotational levels. We then sum the fractional populations in the levels that we have observed (i.e., $J_{u}=2,3,5,7,8$, 9,11 , and 15) which provides the fractional population in all observed levels $\left(f_{\text {obs }}\right)$. The correction factor which we apply to obtain the population in all levels is then given by $f_{c}=1 / f_{\text {obs }}$. Based on this calculation, Figure 3 shows that if we are probing gas with $10 \mathrm{~K}<T<400 \mathrm{~K}$, then simply summing up the integrated emission of $\mathrm{C}^{18} \mathrm{O}$ detections provides an estimate of the total $\mathrm{C}^{18} \mathrm{O}$ column to within a factor of $<2$. The correction factor at $T>100 \mathrm{~K}$ is higher for higher density gas since the combination of high temperature and density means that states higher than $J=15$ can become significantly populated. At lower temperatures, much of the population resides in the $J=0,1$, and 2 states and, therefore, the high- $J$ states that we have observed do not significantly contribute to the overall population. Table 3 shows the summed column density in the observed states $\left(\sum N_{u}\right)$, the correction factor $\left(f_{c}\right)$, and the total $\mathrm{C}^{18} \mathrm{O}$ column density $\left(N_{\mathrm{tot}}\right)$. 
Table 3

$\mathrm{C}^{18} \mathrm{O}$ Column Density toward Orion KL

\begin{tabular}{|c|c|c|c|c|c|}
\hline Component & $\begin{array}{c}\sum N_{u} \mathrm{C}^{18} \mathrm{O} \\
\left(\mathrm{cm}^{-2}\right)\end{array}$ & $f_{c}$ & $N_{\text {tot }} \mathrm{C}^{1}$ & $\begin{array}{l}{ }^{8} \mathrm{O} \pm \text { error } \\
\left.\mathrm{m}^{-2}\right)\end{array}$ & $\begin{array}{l}N_{\text {tot }} \mathrm{H}_{2} \\
\left(\mathrm{~cm}^{-2}\right)\end{array}$ \\
\hline Extended Ridge & $7.85 \times 10^{15}$ & 1.81 & $1.4 \times 10^{16}$ & $\begin{array}{l}+2.4 \times 10^{15} \\
-4.0 \times 10^{14}\end{array}$ & $7.1 \times 10^{22}$ \\
\hline Outflow/Plateau & $2.12 \times 10^{16}$ & 1.67 & $3.5 \times 10^{16}$ & $\begin{array}{l}+6.3 \times 10^{15} \\
-1.2 \times 10^{15}\end{array}$ & $1.8 \times 10^{23}$ \\
\hline Compact Ridge & $1.29 \times 10^{16}$ & 1.67 & $2.2 \times 10^{16}$ & $\begin{array}{l}+8.2 \times 10^{15} \\
-1.9 \times 10^{15}\end{array}$ & $1.1 \times 10^{23}$ \\
\hline Hot Core & $3.67 \times 10^{16}$ & 1.69 & $6.2 \times 10^{16}$ & $\begin{array}{l}+1.8 \times 10^{16} \\
-4.9 \times 10^{15}\end{array}$ & $3.1 \times 10^{23}$ \\
\hline
\end{tabular}

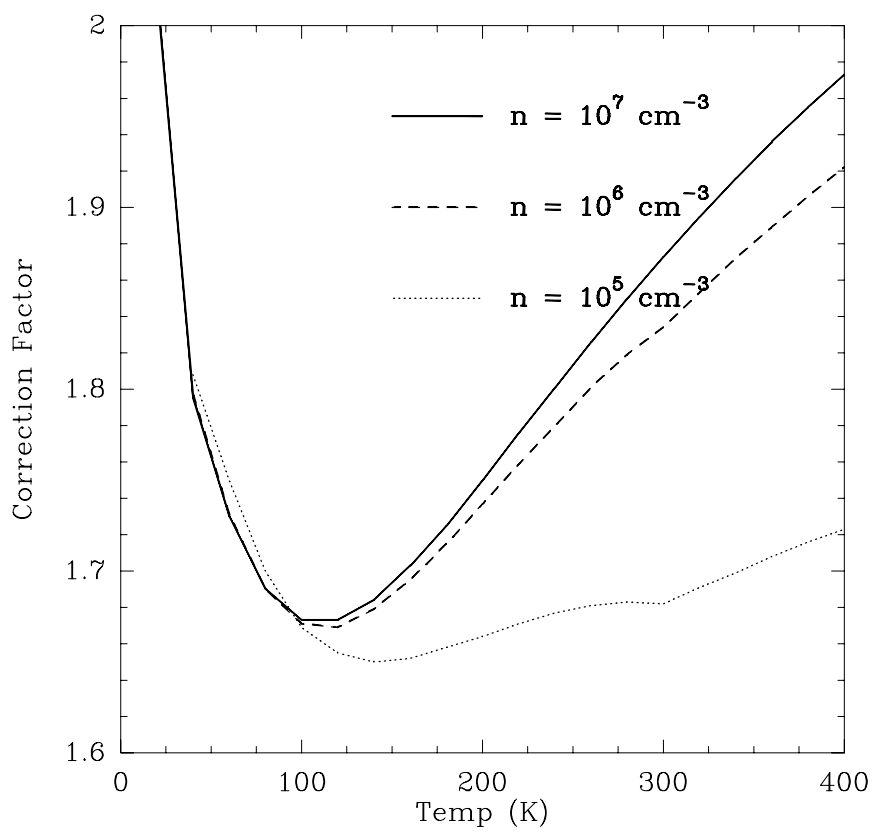

Figure 3. Correction factor for missing population assuming you have observed $\mathrm{C}^{18} \mathrm{O}$ transitions accessible to Herschel/HIFI $\left(J_{u}=5,7,8,9,10,11,15\right)$, as well as $J_{u}=2$ and 3 from the CSO, as a function of temperature.

Scaling the $\mathrm{C}^{18} \mathrm{O}$ column densities in Table 3 by the canonical $\mathrm{CO}: \mathrm{C}^{18} \mathrm{O}$ abundance ratio of 500 , we find that our $\mathrm{CO}$ column density in the Extended Ridge is approximately 2-3 times lower than that tabulated in Blake et al. (1987). Since Blake et al. do not provide a separate column density for the Compact Ridge, it may be that their tabulated $\mathrm{CO}$ column density incorporates blended emission from the Compact Ridge as well. The combination of the Extended and Compact Ridge column densities listed in Table 3 results in a CO column density of $1.8 \times 10^{19} \mathrm{~cm}^{-2}$ which compares favorably with Blake et al.'s $\mathrm{CO}$ column density of $1.5 \times 10^{19} \mathrm{~cm}^{-2}$.

Our calculations of the CO column density in the Outflow/Plateau and Hot Core, however, are $\sim 3$ times larger than those listed in Blake et al. (1987). This could be a result of the fact that Blake et al. calculated the total CO column density based on only two low-energy transitions, $\mathrm{C}^{18} \mathrm{O} 2-1$ and $\mathrm{C}^{17} \mathrm{O} 2-1$. Since the gas temperatures in the Outflow/Plateau and Hot Core are $>100 \mathrm{~K}$, a calculation of the total column density based on only these low-energy lines may severely underestimate the actual amount of hot gas present. Comito et al. (2005) observed the higher energy 8-7 transition of $\mathrm{C}^{18} \mathrm{O}(877 \mathrm{GHz})$ and found a rough estimate of $1.7 \times 10^{17} \mathrm{~cm}^{-2}$. While they assume that the $\mathrm{C}^{18} \mathrm{O} 8-7$ emission comes mainly from the Hot Core and make no attempt to separate the contributions from the different physical components, their total column density compares favorably to the sum of the four components listed in Column 4 of Table 3 (i.e., $1.3 \times 10^{17} \mathrm{~cm}^{-2}$ ). In the last column of Table 3 we have scaled the $\mathrm{C}^{18} \mathrm{O}$ column density up by a factor of $500 \times 10^{4}$ to obtain the canonical $\mathrm{H}_{2}$ column density in these regions.

Finally, it should be noted that the total $\mathrm{C}^{18} \mathrm{O}$ column densities determined from the LTE and non-LTE modeling were identical to within 5\%-10\%. This suggests that, for the high densities in the various Orion KL components, LTE is a reasonable assumption. Thus, since the $\mathrm{C}^{17} \mathrm{O}$ collision rates have not yet been included within the CASSIS implementation of RADEX, we present only the LTE results for consistency.

Figure 4 shows the comparison between an LTE model and the line intensities $\left(T_{R}^{*}\right)$ extracted for each of the line-of-sight components (see Figure 2). The LTE model for each component was produced using the $\mathrm{C}^{18} \mathrm{O}$ column densities listed in Table 3 and the physical conditions listed in Table 2. The "dip" between $J_{\text {upper }}=3$ and 5 in the Compact Ridge and the Hot Core is a result of switching from the CSO (a $10.4 \mathrm{~m}$ telescope) to Herschel (a $3.5 \mathrm{~m}$ telescope) in our observations of these warm, compact objects. Thus, between $J_{\text {upper }}=3$ and 5 there is a large change in the beam filling factor. Since the Extended Ridge and Outflow/Plateau are extended objects, beam filling factor is less of an issue. It is quite remarkable that, except for the Outflow/Plateau, a simple LTE model fits the observations over a wide range of transitions and energies. The relatively poor fit to the Outflow/Plateau may be a result of the allocation of intensity to the various line-of-sight components. We clearly underestimate the $J=3-2$ component which may be a result of the relatively low signal-to-noise ratio $(\mathrm{S} / \mathrm{N})$ in the $J=3-2$ spectrum that may be masking the $J=3-2$ emission from the outflow. In the mid- $J$ states (i.e., $J=7-11$ ), we seem to be attributing too much emission to the outflow which simultaneously underestimates the emission coming from the Compact Ridge and Hot Core. These Outflow/Plateau intensities, however, were required to fit the extended "line-wing" emission seen in Figure 2 and, thus, cannot be reduced significantly without reducing the quality of the overall fits. One possible solution, however, would be to model the high-velocity and the lowervelocity components separately-effectively using five line-ofsight components.

\subsection{Modeling the $C^{17} \mathrm{O}$ Line Emission}

Using the same technique applied to the $\mathrm{C}^{18} \mathrm{O}$ data, we have also fit the $\mathrm{C}^{17} \mathrm{O}$ transitions (Figures 5 and 6) and calculated the total $\mathrm{C}^{17} \mathrm{O}$ column densities in each of the four physical components in Orion KL, as well as the $\mathrm{C}^{18} \mathrm{O} / \mathrm{C}^{17} \mathrm{O}$ abundance ratios (see Table 4). The correction factors for $\mathrm{C}^{17} \mathrm{O}$ are higher 

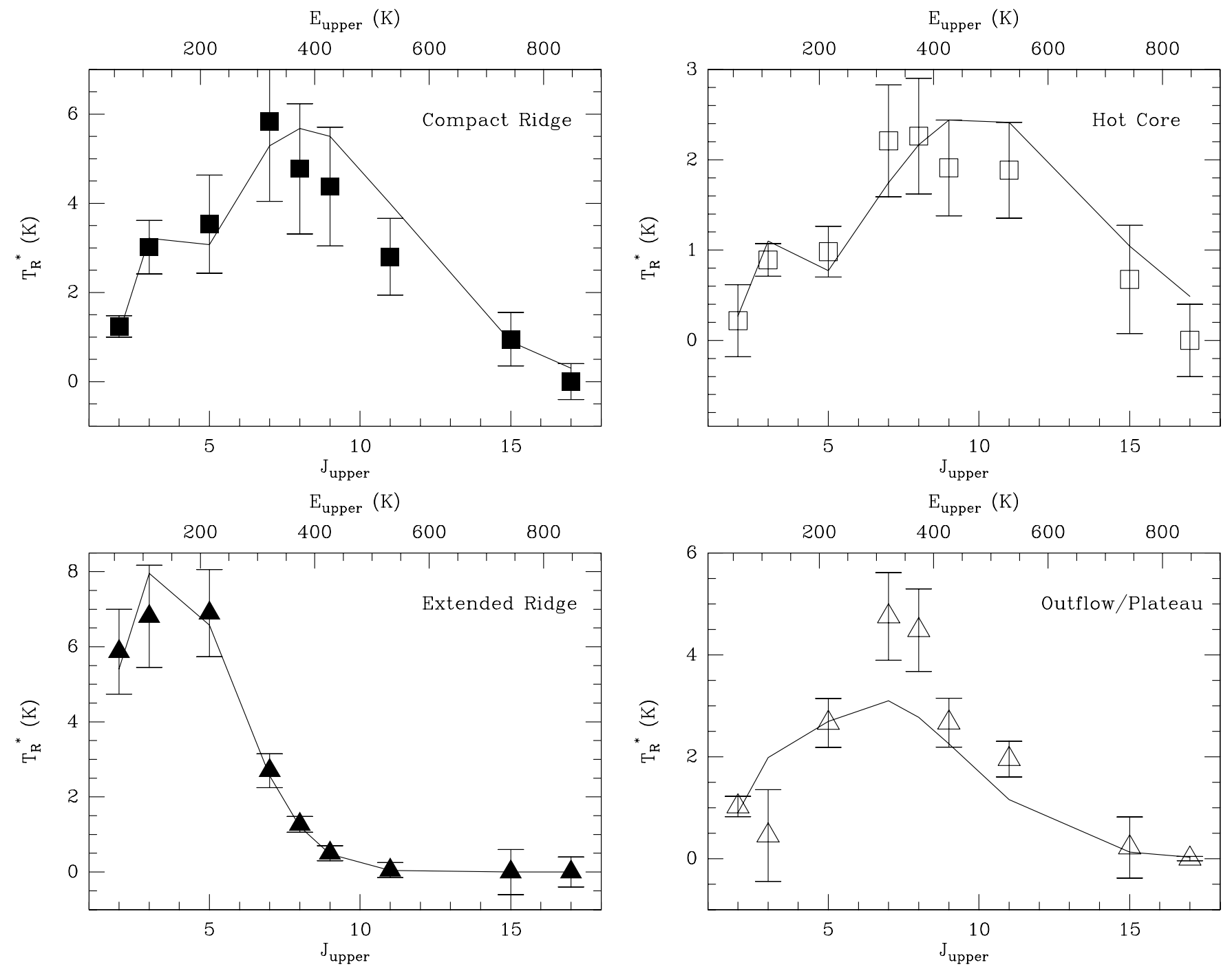

Figure 4. Line intensity $\left(T_{R}^{*}\right)$ vs. $J_{\text {upper }}$ for each of the four line-of-sight components in Orion KL. The solid line shows the results of an LTE model using the $\mathrm{C}^{18} \mathrm{O}$ column density listed in Table 3 and the physical conditions listed in Table 2. The points indicate the best component fits to each of the individual transitions (shown in Figure 2). Error bars reflect the observational uncertainties discussed in Section 3.3 except in cases where these "percentage" uncertainties are smaller than the noise. In these cases, the error bars reflect the $1 \sigma$ rms noise.

Table 4

$\mathrm{C}^{17} \mathrm{O}$ Column Density toward Orion KL

\begin{tabular}{|c|c|c|c|c|}
\hline Component & $\begin{array}{c}\sum N_{u} \mathrm{C}^{17} \mathrm{O} \\
\quad\left(\mathrm{cm}^{-2}\right)\end{array}$ & $f_{c}$ & $\begin{array}{c}N_{\text {tot }} \mathrm{C}^{17} \mathrm{O} \pm \text { error } \\
\left(\mathrm{cm}^{-2}\right)\end{array}$ & $\begin{array}{l}\frac{N_{\text {tot }}\left(\mathrm{C}^{18} \mathrm{O}\right)}{N_{\text {tot }}\left(\mathrm{C}^{17} \mathrm{O}\right)} \pm \text { error } \\
\end{array}$ \\
\hline Extended Ridge & $1.53 \times 10^{15}$ & 4.04 & 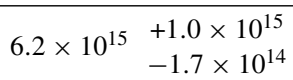 & $2.3_{-0.5}^{+0.5}$ \\
\hline Outflow/Plateau & $7.52 \times 10^{15}$ & 2.79 & $2.1 \times 10^{16} \begin{array}{l}+3.7 \times 10^{15} \\
-7.3 \times 10^{14}\end{array}$ & $1.7_{-0.5}^{+0.4}$ \\
\hline Compact Ridge & $2.16 \times 10^{15}$ & 2.50 & $\begin{array}{ll}5.4 \times 10^{15} & +2.0 \times 10^{15} \\
-4.7 \times 10^{14}\end{array}$ & $4.1_{-1.3}^{+2.1}$ \\
\hline Hot Core & $8.97 \times 10^{15}$ & 2.30 & $\begin{array}{rr}2.1 \times 10^{16} & +6.0 \times 10^{15} \\
& -1.6 \times 10^{15} \\
\end{array}$ & $3.0_{-1.1}^{+1.2}$ \\
\hline
\end{tabular}

than for $\mathrm{C}^{18} \mathrm{O}$ since, without the ground-based $3-2$ observations, we have one less observed transition. This is most noticeable in the Extended Ridge component because, at $40 \mathrm{~K}$, the bulk of the population will be in the states with $J<7$.

While the $\mathrm{C}^{17} \mathrm{O} 7-6$ spectrum (Figure 6, top left panel) is seemingly simple to analyze, it is actually the most problematic of all the spectral lines in this study due to the presence of strong $\mathrm{H}_{2} \mathrm{CO}$ emission which must be modeled in order to obtain the true intensity of the $\mathrm{C}^{17} \mathrm{O}$ lines. Comito et al. (2005) find ortho$\mathrm{H}_{2} \mathrm{CO}$ column densities in the Hot Core and Compact Ridge of $2 \times 10^{15}$. The transition at $786.28 \mathrm{GHz}$, however, is the $11_{0,11}-10_{0,10}$, which corresponds to para $\mathrm{H}_{2} \mathrm{CO}$. Therefore, we reduce the model column densities by a factor of three (the ortho-to-para ratio) and then model the $\mathrm{C}^{17} \mathrm{O}$ line intensities concomitantly with the para- $\mathrm{H}_{2} \mathrm{CO}$ lines. In the $\mathrm{C}^{17} \mathrm{O} 8-7$ spectrum (Figure 6, top right panel) the additional spectral 


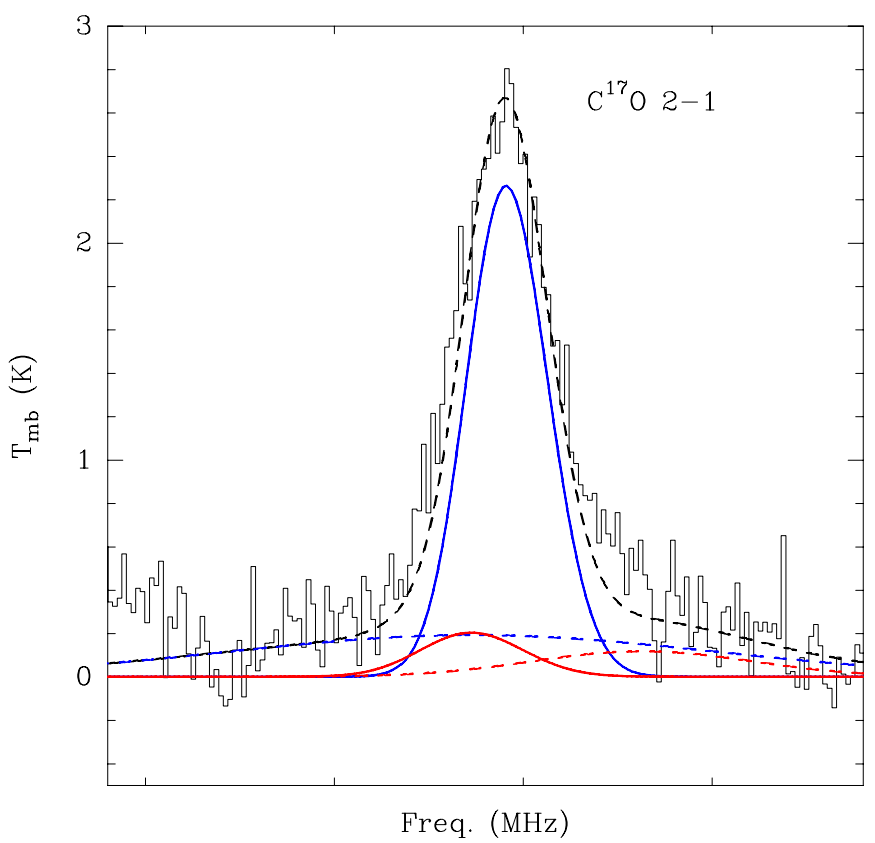

Figure 5. $\mathrm{C}^{17} \mathrm{O} 2-1(\sim 224.714 \mathrm{GHz})$ spectrum from the CSO (histogram) and results of LTE modeling. Results are shown for the Extended Ridge (solid blue), Outflow/Plateau (dashed blue), Compact Ridge (solid red), Hot Core (dashed red), and for the sum of all four components (dashed black).

(A color version of this figure is available in the online journal.)

feature at $898.5 \mathrm{GHz}$ is likely a blend of $\mathrm{S}_{2} \mathrm{O}, v=0$ lines, whereas the feature at $898.55 \mathrm{GHz}$ may be due to emission from NS. The only transitions listed in the catalogs that fall at the $\mathrm{C}^{17} \mathrm{O} 8-7$ frequency and have an energy less than $700 \mathrm{~K}$ belong to $\mathrm{PO}_{2}$ and $\mathrm{HCCCH}_{2} \mathrm{OH}$. However, neither of these appear to contribute significantly to the observed line profile. For $\mathrm{C}^{17} \mathrm{O} 9-8$ (Figure 6, middle left panel), the extra features in the spectrum, as well as the asymmetric shape of the main feature, are due to numerous $\mathrm{CH}_{3} \mathrm{OH}$ transitions. We have modeled the $\mathrm{CH}_{3} \mathrm{OH}$ as arising from the compact ridge with column densities of $2 \times 10^{17} \mathrm{~cm}^{-2}$ which allows us to fit the blended $\mathrm{C}^{17} \mathrm{O}$ and $\mathrm{CH}_{3} \mathrm{OH}$ lines as well as the two lines on the right side of the figure. Although this is higher than the $5 \times 10^{16} \mathrm{~cm}^{-2}$ found by Comito et al. (2005), we still under-fit the latter two features. However, raising the $\mathrm{CH}_{3} \mathrm{OH}$ column above $2 \times 10^{17} \mathrm{~cm}^{-2}$ results in too much emission in the blended components, and requires us to reduce the $\mathrm{C}^{17} \mathrm{O}$ column densities to unreasonably low values. Thus, we choose $2 \times 10^{17} \mathrm{~cm}^{-2}$ as a reasonable compromise for the methanol column density. In the $\mathrm{C}^{17} \mathrm{O} 10-9$ panel (Figure 6, middle right panel) there are no additional molecular transitions that likely contribute significantly to the observed line profile. In the $\mathrm{C}^{17} \mathrm{O} 11-10$ panel (Figure 6, bottom left panel) the small "hump" to the left (near $1234 \mathrm{GHz}$ ) may be a contribution from a vibrationally excited $\mathrm{SO}_{2}$ line or, possibly, four hyperfine lines of $\mathrm{H}_{2} \mathrm{Cl}^{+}$. The $\mathrm{C}^{17} \mathrm{O} 13-12$ emission (Figure 6, bottom left panel) is only an upper limit since no significant emission was detected. If we eliminate this line from our analysis, we change the total $\mathrm{C}^{17} \mathrm{O}$ column density by $<3 \%$ in the hot core, $<2 \%$ in Compact Extended ridge, and $<1 \%$ in the Outflow/Plateau and Extended Ridge.

Table 4 also lists the resultant $\mathrm{C}^{18} \mathrm{O} / \mathrm{C}^{17} \mathrm{O}$ abundance ratio (hereafter referred to as $R$ ) and shows that $R$ varies in Orion: from 1.7 in the Outflow/Plateau, 2.3 in the Extended Ridge, 3.0 in the Hot Core, to 4.1 in the Compact Ridge. While the terrestrial ratio is 5.5 (Heikkilä et al. 1998), there is some discrepancy in the interstellar values. For example, Penzias (1981) measured $R \approx 3-4$ in a number of giant molecular clouds (e.g., SgrA, SgrB, W33, W51, M17, DR21, Ori A, NGC 2024, W3, NGC 7538), Wouterloot et al. (2008) found $R=4.1$ in $\rho$ Ophiuchus, Wilson et al. (1981) finds $R=2.9$ in three dark clouds, and Ladd (2004) finds $R=2.8$ in Taurus.

In the latter paper, Ladd (2004) has assumed a model in which two distinct phases exist: an inner region in which both the $\mathrm{C}^{18} \mathrm{O}$ and $\mathrm{C}^{17} \mathrm{O}$ are well shielded from ultraviolet radiation, and an outer "sheath" in which the $\mathrm{C}^{17} \mathrm{O}$ has been selectively dissociated. Thus, in this model, higher values of $R$ may be found in regions with stronger UV radiation fields. This may help explain the variable ratios in our data since the Hot Core and Compact Ridge have higher temperatures, likely due to their more intimate association with the embedded sources in the vicinity of the Hot Core (such as the UCHII regions "I" and "L"-Menten \& Reid 1995; Gezari et al. 1998) and, thus, may be more directly impacted by the radiation field. Note, however, that given the size of the uncertainty in $R$, no definitive statement can be made.

\subsection{Observational Uncertainty}

Because of the use of a large number of rotational transitions that span the range of excitation (from weak-to strong-to weak), our estimates of the total column and isotopic ratios represent the best effort to date to derive these fundamental quantities. It is thus worthwhile to discuss the various sources of uncertainty that are present in our data or are placed in the derivation due to our assumptions. A primary uncertainty inherent in millimeterand submillimeter-wave data is the telescope efficiency which corrects for telescopic and atmospheric losses. As a space-borne platform Herschel minimizes many of these uncertainties and the absolute calibration error is likely below $\sim 15 \%$.

Our assumptions regarding excitation provide a large source of uncertainty. As discussed earlier, we have explored both LTE and non-LTE analyses for our data and find only a small difference of 5\%-10\%. This is important as we have assumed optically thin emission for our LTE analysis. However, from our non-LTE analysis, we have found that all $\mathrm{C}^{18} \mathrm{O}$ and $\mathrm{C}^{17} \mathrm{O}$ lines in all components are optically thin. In fact, the largest opacity is 0.33 and is found in the $\mathrm{C}^{18} \mathrm{O} J=5-4$ transition in the Extended Ridge. All other components and lines have lower opacities. In particular, the Compact Ridge and Hot Core both have $\tau \leqslant 0.15$ for all transitions of $\mathrm{C}^{18} \mathrm{O}$, and the Outflow/Plateau has $\tau<0.1$ (again for all $\mathrm{C}^{18} \mathrm{O}$ transitions). Thus, the small change between the LTE and non-LTE results are probably due to the fact that the transitions are optically thin and, given the densities in Orion $\mathrm{KL}$, most transitions are likely in LTE or at least close to it.

The largest source of uncertainty in our estimates of the total column is introduced by the uniform source size for a given spatial/velocity component. If we decrease the size of all components by $20 \%$, we expect to have to increase the column densities in order to appropriately refit the spectral line profiles. In fact, the column density needed to fit the Extended Ridge does not change, due to the fact that the reduced size of this source is still larger than the Herschel beam at all frequencies. The column density of the Outflow/Plateau increases by only $6 \%$, again due to the fact that the reduced source size is still larger than the Herschel beam. The Compact Ridge and Hot Core column densities, however, increase by $34 \%$ and $24 \%$ since their extent is smaller than the beam at many of the observed frequencies. If we increase the size of each component by $20 \%$, again, the column density in the Extended Ridge does not change. However, the column densities in the Outflow/Plateau, 

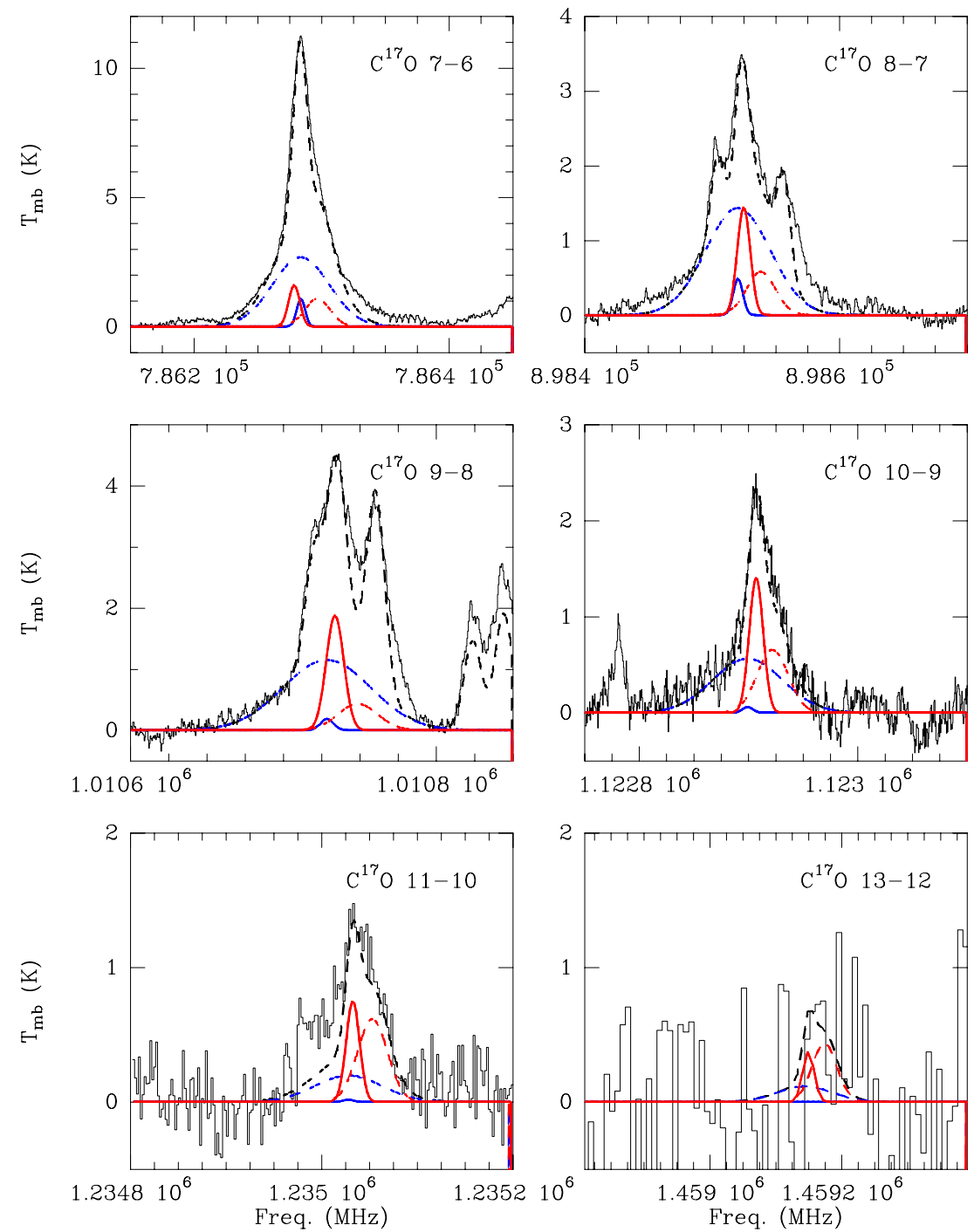

Figure 6. Spectra (histograms) and results of LTE modeling of the $\mathrm{C}^{17} \mathrm{O} J=7-6(\sim 786.280 \mathrm{GHz}), J=8-7(\sim 898.522 \mathrm{GHz}), J=9-8(\sim 1010.731 \mathrm{GHz}), J=10-9$ ( $\sim 1122.902 \mathrm{GHz}), J=11-10(\sim 1235.031 \mathrm{GHz})$, and $J=13-12(\sim 1459.148 \mathrm{GHz})$ transitions in Orion $\mathrm{KL}$ (frequencies are approximate since $\mathrm{C}^{17} \mathrm{O}$ has hyperfine components). Results are shown for the Extended Ridge (solid blue), Outflow/Plateau (dashed blue), Compact Ridge (solid red), Hot Core (dashed red), and for the sum of all four components (dashed black) including emission from species other than $\mathrm{C}^{17} \mathrm{O}$.

(A color version of this figure is available in the online journal.)

Compact Ridge, and Hot Core need to be decreased by $10 \%$, $16 \%$, and $21 \%$, respectively.

The total error budget must, therefore, include the $15 \%$ HIFI calibration error, a $\sim 7.5 \%$ error introduced by using LTE models, and the column density errors introduced by $20 \%$ variations in source size. The uncertainties presented in Tables 3 and 4 , thus, reflect these errors added in quadrature.

\section{CONCLUSIONS}

Using the HIFI instrument on board the Herschel Space Observatory, we have observed $\mathrm{C}^{18} \mathrm{O} 5-4,7-6,8-7,9-8$, $11-10,15-14$, and 17-16 and $\mathrm{C}^{17} \mathrm{O} 7-6,8-7,9-8,10-9,11-10$, 13-12, 15-14, and 16-15. Combined with $\mathrm{C}^{18} \mathrm{O} 3-2$ and 2-1 and $\mathrm{C}^{17} \mathrm{O} 2-1$ observations from the $\mathrm{CSO}$, we have calculated the total $\mathrm{C}^{18} \mathrm{O}$ and $\mathrm{C}^{17} \mathrm{O}$ column densities in the four major line-of-sight components in Orion KL: the Extended Ridge, the Outflow/Plateau, the Compact Ridge, and the Hot Core by fitting model line profiles to each transition. The model fits provide the column density in each upper state which are then summed to produce the total column density (after correcting by the population fraction in the unobserved states). With the large number of transitions available to HIFI, the correction factors are less than a factor of two in gas with temperatures $>100 \mathrm{~K}$. Thus, this method provides a more accurate calculation of the total column density in warm gas than can be obtained by observing only a few, lower energy transitions accessible from the ground.

Using this method, the total $\mathrm{C}^{18} \mathrm{O}$ column densities in the Extended Ridge, Outflow/Plateau, Compact Ridge, and Hot Core components of Orion KL is $1.4 \times 10^{16} \mathrm{~cm}^{-2}, 3.5 \times$ $10^{16} \mathrm{~cm}^{-2}, 2.2 \times 10^{16} \mathrm{~cm}^{-2}$, and $6.2 \times 10^{16} \mathrm{~cm}^{-2}$, respectively. These total columns will be useful to derive abundances for numerous species in this prototypical massive star-forming region. In addition, the total column can be used in studies of dust emission to constrain the overall emission along the line of sight and hence constrain the combined effects of dust column, thermal gradients, and mass opacity corrections.

We also find that the $\mathrm{C}^{18} \mathrm{O} / \mathrm{C}^{17} \mathrm{O}$ abundance ratio varies from 1.7 in the Outflow/Plateau, 2.3 in the Extended Ridge, 3.0 in the Hot Core, and to 4.1 in the Compact Ridge. This suggests a 
model in which regions with higher ultraviolet radiation fields may be selectively photodissociating $\mathrm{C}^{17} \mathrm{O}$.

HIFI has been designed and built by a consortium of institutes and university departments from across Europe, Canada, and the United States under the leadership of SRON Netherlands Institute for Space Research, Groningen, The Netherlands, and with major contributions from Germany, France, and the US. Consortium members are Canada: CSA, U.Waterloo; France: CESR, LAB, LERMA, IRAM; Germany: KOSMA, MPIfR, MPS; Ireland, NUI Maynooth; Italy: ASI, IFSI-INAF, Osservatorio Astrofisico di Arcetri-INAF; The Netherlands: SRON, TUD; Poland: CAMK, CBK; Spain: Observatorio Astronmico Nacional (IGN), Centro de Astrobiologa (CSIC-INTA); Sweden: Chalmers University of Technology-MC2, RSS \& GARD; Onsala Space Observatory; Swedish National Space Board, Stockholm University-Stockholm Observatory; Switzerland: ETH Zurich, FHNW; USA: Caltech, JPL, NHSC. Support for this work was provided, in part, by a National Sciences and Engineering Research Council of Canada (NSERC) grant to R. Plume, by the National Science Foundation grant AST0838261 to the Caltech Submillimeter Observatory, and by NASA through an award issued by JPL/Caltech.

Facility: Herschel, CSO

\section{REFERENCES}

Acharyya, K., Fuchs, G. W., Fraser, H. J., van Dishoeck, E. F., \& Linnartz, H. 2007, A\&A, 466, 1005
Bergin, E. A., Goldsmith, P. F., Snell, R. L., \& Ungerechts, H. 1994, ApJ, 431 674

Bergin, E. A., Phillips, T. G., Comito, C., et al. 2010, A\&A, 521, L20

Bergin, E. A., \& Tafalla, M. 2007, ARA\&A, 45, 339

Blake, G. A., Sutton, E. C., Masson, C. R., \& Phillips, T. G. 1987, ApJ, 315 , 621

Comito, C., Schilke, P., Phillips, T. G., et al. 2005, ApJS, 156, 127

Genzel, R., \& Stutzki, J. 1989, ARA\&A, 27, 41

Gezari, D. Y., Backman, D. E., \& Werner, M. W. 1998, ApJ, 509, 283

Goldsmith, P. F., Bergin, E. A., \& Lis, D. C. 1997, ApJ, 491, 615

Heikkilä, A., Johansson, L. E. B., \& Olofsson, H. 1998, A\&A, 332, 493

Ladd, E. F. 2004, ApJ, 610, 320

Maret, S., Hily-Blant, P., Pety, J., Bardeau, S., \& Reynier, E. 2011, A\&A, 526, A47

Menten, K. M., \& Reid, M. J. 1995, ApJ, 445, L157

Müller, H. S. P., Schlöder, F., Stutzki, J., \& Winnewisser, G. 2005, J. Mol. Struct., 742, 215

Müller, H. S. P., Thorwirth, S., Roth, D. A., \& Winnewisser, G. 2001, A\&A, 370, L49

Penzias, A. A. 1981, ApJ, 249, 518

Persson, C. M., Olofsson, A. O. H., Koning, N., et al. 2007, A\&A, 476, 807

Pickett, H. M., Poynter, R. L., Cohen, E. A., et al. 1998, J. Quant. Spectrosc. Radiat. Transfer, 60, 883

Plambeck, R. L., Wright, M. C. H., Welch, W. J., et al. 1982, ApJ, 259, 617

Plume, R., Jaffe, D. T., Evans, N. J., II, Martin-Pintado, J., \& Gomez-Gonzalez, J. 1997, ApJ, 476, 730

Shirley, Y. L., Huard, T. L., Pontoppidian, K. M., et al. 2011, ApJ, 728, 143

Van der Tak, F. F. S., Black, J. H., Schier, F. L., Jansen, D. J., \& van Dishoeck, E. F. $2007, A \& A, 468,627$

Wilson, R. W., Langer, W. D., \& Goldsmith, P. F. 1981, ApJ, 243, L47

Wouterloot, J. G. A., Brand, J., \& Henkel, C. 2008, A\&A, 430, 549

Wright, M. C. H., Plambeck, R. L., Vogel, S. N., Ho, P. T. P., \& Welch, W. J. 1983, ApJ, 267, L41 